\title{
Cédric de Guido, Marcel Schwob, du journal au recueil
}

Paris, Classiques Garnier, 2015, 414 p.

Pauline Boch

\section{OpenEdition \\ Journals}

Édition électronique

URL : http://journals.openedition.org/recherchestravaux/929

DOI : 10.4000/recherchestravaux.929

ISSN : 1969-6434

Éditeur

UGA Éditions/Université Grenoble Alpes

Édition imprimée

ISBN : 978-2-37747-014-3

ISSN : 0151-1874

\section{Référence électronique}

Pauline Boch, « Cédric de Guido, Marcel Schwob, du journal au recueil », Recherches \& Travaux [En ligne], 91 | 2017, mis en ligne le 01 octobre 2017, consulté le 25 septembre 2020. URL : http://

journals.openedition.org/recherchestravaux/929; DOI : https://doi.org/10.4000/recherchestravaux. 929

Ce document a été généré automatiquement le 25 septembre 2020.

(c) Recherches \& Travaux 


\section{Cédric de Guido, Marcel Schwob, du journal au recueil}

Paris, Classiques Garnier, 2015, 414 p.

\section{Pauline Boch}

\section{RÉFÉRENCE}

Cédric de Guido, Marcel Schwob, du journal au recueil, Paris, Classiques Garnier, 2015,

$414 \mathrm{p}$.

Avec Marcel Schwob, du journal au recueil, Cédric de Guido invite à repenser l'œuvre de Marcel Schwob en faisant l'hypothèse d'une littérature très largement conçue pour la presse - et en ce sens déterminée par certains de ses impératifs (brièveté, actualité, « rubricité », etc.) -, mais aussi héritière d'une tradition philologique qui fait la part belle à la démonstration érudite. Par-delà son statut incontestable de conteur, c'est à sa figure d'écrivain-journaliste que Cédric de Guido tente ainsi de nous rendre attentifs. Ayant publié l'ensemble de ses textes, de facture immuablement brève, dans des quotidiens tels que L'Écho de Paris, Le Journal ou Le Phare de la Loire, avant de les réunir dans des recueils qui ont défini pour une large part leur appartenance aux genres fictionnels, Marcel Schwob n'a donc pu, comme le fait remarquer l'auteur, s'affranchir complètement des codes de l'écriture journalistique, cette «matrice» définie par Marie-Ève Thérenty dans son ouvrage de 2007, La littérature au quotidien. Poétiques journalistiques au XIX $X^{e}$ siècle, auquel le critique se réfère volontiers et qui a inspiré dernièrement, tout comme les recherches menées par Alain Vaillant, une série de travaux consacrés à l'histoire et à la poétique de la presse littéraire de ce siècle. Si homogénéité il y a - dans une œuvre qui résiste à la synthèse et à la généralisation, en ce qu'elle présente une mosaïque de textes génériquement et stylistiquement contrastés -, celle-ci s'explique essentiellement par la manière singulière qu'a l'auteur de composer avec les exigences de la presse, recourant à des techniques poétiques de « fictionnalisation du savoir » qui tendent à brouiller la frontière séparant la réalité de 
la fiction: c'est là la thèse défendue par l'auteur, avec une constance imperturbable, dans un ouvrage tout à la fois imposant et minutieux.

2 Aussi cette enquête invite-t-elle d'abord à envisager l'œuvre du point de vue de la «matrice » journalistique, plutôt que de celui de l'effet de recueils, constitués pour la plupart a posteriori, et modifiant tout à la fois l'horizon de réception des textes, leur affiliation générique et leur dynamique polyphonique. Pour Cédric de Guido, la mise en recueil ne peut se targuer de sceller le destin fictionnel des écrits de Marcel Schwob au détriment de leur statut initialement hybride, à la croisée du réel et de l'imaginaire, métissage de la chronique et de la fiction. Cédric de Guido propose donc une taxinomie générique nouvelle, «point de départ d'une réflexion qui en montre les limites » (p. 36) tout autant que la porosité, mais qui cherche à la fois à interroger la place du texte sur la page du journal et à mettre au jour son ambition paradoxale de "fictionnaliser " l'actualité (et le réel en général) : les textes seront ainsi examinés en tant que critiques littéraires ou linguistiques et chroniques d'actualité, en tant que chroniques inactuelles, et finalement, en tant que contes. C'est donc à partir de cette réorganisation générique que le critique construit et ordonne les grandes lignes de sa réflexion dans un ouvrage somme, agencé en triptyque, dont l'ambition est de revisiter l'ensemble de la production de Marcel Schwob à travers le prisme des exigences journalistiques.

3 Dans une première partie intitulée "Marcel Schwob, "polygraphe de la modernité" ", Cédric de Guido interroge des textes qui présentent le plus de traits communs avec la chronique journalistique traditionnelle. Il s'agit de montrer que la méthode critique adoptée par Schwob dans ses multiples études philologiques et littéraires, dans ses traductions, ses «Notes sur Paris » (qui, selon le critique, peuvent autant être lues comme des fictions que comme des chroniques à caractère ethnologique), ainsi que dans ses préfaces, contribue à élargir la définition du genre : protéiforme, la chronique peut à la fois se targuer d'un discours documentaire (épaisseur historique ou commentaire de l'actualité) et arborer un caractère intemporel. Baptisée « archéologie du savoir » (qui ne doit pas être confondue avec la méthode d'analyse proposée par Michel Foucault) en ce qu'elle adopte le ton d'une neutralité objective et opte pour une approche diachronique de la langue et de la littérature, cette méthode critique, héritière de l'érudition philologique, soustrait les textes de fiction au risque de l'anecdote et de la péremption, écueils emblématiques de la publication journalistique :

Là où le journaliste cherchera à mettre en scène sa propre crédibilité de témoin, ou de spécialiste de l'étude de l'argot, ou de grand arpenteur des faubourgs, etc., l'écrivain cherche à "vraisemblabiliser" la présence des informations qu'il souhaite dispenser dans le discours d'un personnage précis à un moment précis de la fiction: crédibilité et vraisemblance n'étant [...] que deux aspects d'un même problème, celui de la présence du savoir dans le texte. » (p. 103-104)

Mais selon Cédric de Guido, cette "fictionnalisation " d'un savoir semé au fil des publications constitue également «une parade patiemment chorégraphiée dans l'intention de construire un ethos crédible» (p. 85) de l'écrivain-journaliste. Cette érudition, Schwob la destine principalement à un public averti, comme en témoignent certaines de ses "Lettres parisiennes", sortes de chroniques moralistes publiées dans le Phare de la Loire. Dans ce quotidien nantais, consommé par un lectorat provincial éloigné des préoccupations de la capitale, ces chroniques doivent, pour être comprises en leur qualité de commentaire critique et ironique, se lire dans une actualité parisienne qui n'est connue que des « happy few ». Possédant l'art de la chronique dite 
«factuelle », Schwob peut s'essayer à la «fiction d'actualité ", genre hybride évoqué par Marie-Ève Thérenty et défini comme une " fiction brève dont l'intrigue se trouve dans la même "actualité" que le reste du périodique » ou comme « écriture fictionnelle de la contemporanéité » (p. 104).

C'est le cas de plusieurs textes de Cœur double, envisagés jusque-là comme des contes (parce que leur mise en recueil invitait à opérer une dichotomie « artificielle » entre chronique et fiction), mais dont l'ancrage et le soubassement savant justifient une reformulation générique. Bien qu'il fasse usage d'une taxinomie nouvelle (qui ajoute parfois, il faut le dire, à la confusion générale régnante lorsque l'on cherche à déterminer les genres pratiqués par Schwob), Cédric de Guido conclut son examen de façon nuancée, mettant en évidence les limites de sa propre typologie, et évoquant, à titre d'exemple, l'indétermination générique dans laquelle peuvent se trouver des écrits tels que les "Lettres à Valmont ", « fictions critiques d'actualité ». Sous leurs airs évidents de pastiches (des Liaisons dangereuses), ces textes n'en sont pas moins selon lui des chroniques épistolaires subtilement connectées à l'actualité mondaine, tout autant que des chroniques faisant écho à une critique plus générale de l'institutionnalisation de la littérature. Schwob, qui, "par référence directe et/ou par fictionnalisation du reportage»(p.140), place l'actualité au centre d'un nombre imposant de textes, redéfinit ainsi le rôle de l'écrivain-journaliste : en travaillant minutieusement la forme, le style et les références de ses chroniques, il parvient ainsi à les soustraire à la naturelle péremption de leur contenu.

Pour une grande part cependant, l'œuvre de Schwob comprend des récits étrangers aux préoccupations quotidiennes. Loin de marquer «une transition symétrique du journalisme à la littérature » (p. 146) - car ils empruntent certains des traits de la chronique et continuent de s'adapter aux contraintes de la presse-, ceux-ci s'attachent à dépeindre une forme d'actualité du passé. Ce sont ces chroniques dites "inactuelles", qui composent LaCroisade des enfants, Mimes et Vies imaginaires, auxquelles le critique consacre la deuxième partie de son étude. Laboratoire de la diffusion d'un savoir historique savant, ces textes font bien de Schwob un "chroniqueur érudit ", ainsi que le suggère le titre même de ce développement. À la source de sa réflexion se dessine une question de taille : quel rapport existe-t-il entre la décision de construire un ethos d'écrivain dont le savoir (philologique, historique et littéraire) est incontestable et le choix de l'exhiber sur la page du journal ? Autrement dit, «qu'est-ce qui fonde la crédibilité de l'écriture journalistique dans sa prétention à dire le réel ? » (p. 145). On sait que la problématique de la mimesis a largement occupé la pensée esthétique de Schwob; Cédric de Guido propose ainsi une synthèse extrêmement intéressante du "réalisme » (à ne pas confondre avec l'école proprement dite) que Schwob a cherché à incarner au cours de sa carrière littéraire : enjeu d'une écriture qui, en multipliant les manières d'insérer un discours sur le réel dans le cadre de la fiction, s'est mise en peine de rappeler que l'imaginaire, qui découle avant tout de l'observation de la nature, doit susciter la même confiance que toute description de la réalité.

Dans cette optique, il n'est pas nécessaire que le lecteur saisisse les enjeux de ce savoir, ni que celui-ci soit exactement concordant avec les événements de l'histoire, mais il est essentiel que le lecteur croie en sa vraisemblance et s'en remette à la figure qui l'incarne: l'écrivain-journaliste. Qu'il s'agisse de LaCroisade des enfants, dont la multiplication des narrations au style archaïque et les précisions minutieuses 
participent d'un effet de vraisemblance, des Vies imaginaires, véritable «cabinet de curiosités » où l'anecdote n'a d'autre vertu que celle d'insister sur le caractère unique et intemporel des personnages, ou encore des Mimes, sorte de chronique antique dont la matière historique est fictivement présentée comme actuelle, tous recourent à une technique poétique et elliptique qui «consiste à laisser au lecteur le soin de reconstruire une cohérence historique à partir de détails parcellaires»(p.237) : contrainte de brièveté journalistique qui ne pouvait que convenir à un écrivain méfiant à l'égard de l'exhaustivité, de l'explication et de la logique causale, et qui assure au texte sa pérennité herméneutique. Plutôt que de prendre la forme de symboles (symbolisme) ou de s'insérer dans une chaîne de déterminations (réalisme et naturalisme), les motifs qui composent la savante «marqueterie » schwobienne n'ont d'autres prétentions que de permettre de rattacher ces récits au domaine de l'histoire et d'assurer aux futurs recueils une cohérence formelle: "signes de signes", ils n'offrent d'autre garantie que celle de structurer l'œuvre.

7 Dans la troisième partie de son étude, de Guido fait le constat de deux contradictions fondamentales chez un « Marcel Schwob, myste littéraire » : d'une part, un journaliste qui, publiant dans la presse, «lieu par excellence de la croyance en un langage directement référentiel»(p.325), ne cesse pourtant de mettre le sens à l'épreuve ; d'autre part, un écrivain qui, embrassant l'idée de la relativité sémiologique, ne fait jamais l'impasse sur ce royaume des références qu'est l'érudition. Autrement dit, c'est le lien complexe et ambigu que l'écrivain entretient avec l'artifice et la référentialité qui est au cœur de ce dernier chapitre. Cédric de Guido invite à se méfier d'interpréter la mise en recueil comme une entreprise unificatrice. Le Roi au masque d'or assurerait certes une littérarité « intemporelle » à une mosaïque de textes bigarrés et modifierait leur réception, mais sa cohérence ostensiblement artificielle ne ferait que renforcer la singularité (autonomie sur la page de journal) et l'ambiguïté (brouillage de la frontière entre réalité et fiction) de récits initialement publiés dans la presse. Ce recueil, étudié thématiquement comme texte homogène sous prétexte de "motifs" récurrents (à l'exemple du masque) mimerait, en leur instillant des significations paradoxales et contradictoires, l'éclatement du sens dans le dessein de refléter "l'instabilité sémiologique du réel discontinu » (p. 309). De la même manière, mais de façon encore plus radicale, Le Livre de Monelle, fondé sur une poétique exclusivement formelle, serait une parole mettant en scène son autoréférentialité. Avec Mœurs des diurnales, qui invite à une réflexion sur l'art du " bien écrire " journalistique, à une époque où la médiocrité de la chronique consensuelle tend à dévoyer les principes mêmes de la profession, Schwob fait le pari, au terme d'un parcours qui lui aura permis d'expérimenter jusqu'à la mystification toutes les techniques de fictionnalisation du savoir, d'incarner le paradox "d'un monde qui fait signe mais qui ne renvoie à aucune référence stable » (p. 383). Aussi serait-il bien ce «faussaire de la nature » dont parlait Georges Trembley, et la presse littéraire, le lieu privilégié pour toiser les lois du réel : « La déconstruction par l'auteur de sa propre autorité, la dérision d'un savoir qui risque de devenir pédant, semblent avoir pour fonction de montrer au lecteur cette artificialité du texte, et de lui suggérer une saine vigilance: il s'agit de le faire entrer dans une sorte d'“ère du soupçon". » (p. 383)

8 Marcel Schwob, du journal au recueil reflète donc une entreprise considérable de relecture de l'œuvre d'un écrivain à la pensée cérébrale et rigoureusement complexe, et l'ouvrage aborde l'épineuse question de l'érudition de Marcel Schwob de manière originale, loin des habituelles problématiques intertextuelles. Pour vertigineux qu'il 
soit, le propos n'en demeure pas moins minutieux et d'une grande qualité analytique, passant en revue chaque texte dans le contexte singulier de sa publication journalistique. Mais dans son effort répété - à la limite parfois de la redondance (tant argumentative que formelle) - pour réhabiliter Marcel Schwob dans son statut d'écrivain-journaliste, l'ouvrage court plus d'un risque: perdre le lecteur dans un dédale de considérations typologiques qui tendent à faire oublier que l'inspiration demeure le moteur premier de la création; mais aussi, tomber dans un essentialisme non moins exclusif que celui qu'il prétend justement contourner dans une œuvre génériquement ambiguë. Mais cela n'enlève rien à l'intérêt ni à la qualité de cette étude: d'une part, les études consacrées à Schwob manquaient, il est vrai, d'une réflexion approfondie au sujet de la relation du texte à son support initial, comme d'une reconsidération de l'œuvre « journalistique », injustement écartée au profit d'une production dite plus « littéraire »; d'autre part, il était temps de proposer une réflexion de plus grande ampleur au sujet de la manière singulière qu'a Schwob de concevoir le monde des références, lieu de tous les possibles, qui réconcilie ses masques littéraires d'érudit et de mystificateur : problématique d'une redoutable complexité qui, dans un dessein peut-être un peu moins schématique, aurait pu sous-tendre à elle seule la réflexion de cette étude importante.

\section{AUTEURS}

\section{PAULINE BOCH}

Université de Fribourg (Suisse) 